On-chip single-copy real-time reverse-transcription PCR in isolated picoliter droplets

N. R. Beer, E. Wheeler, L. Lee-Houghton, N. Watkins, S. Nasarabadi, N. Hebert, P. Leung, D. Arnold, C. Bailey, B. Colston

January 4, 2008

Analytical Chemistry 
This document was prepared as an account of work sponsored by an agency of the United States government. Neither the United States government nor Lawrence Livermore National Security, LLC, nor any of their employees makes any warranty, expressed or implied, or assumes any legal liability or responsibility for the accuracy, completeness, or usefulness of any information, apparatus, product, or process disclosed, or represents that its use would not infringe privately owned rights. Reference herein to any specific commercial product, process, or service by trade name, trademark, manufacturer, or otherwise does not necessarily constitute or imply its endorsement, recommendation, or favoring by the United States government or Lawrence Livermore National Security, LLC. The views and opinions of authors expressed herein do not necessarily state or reflect those of the United States government or Lawrence Livermore National Security, LLC, and shall not be used for advertising or product endorsement purposes. 


\title{
On-chip single-copy real-time reverse-transcription PCR in isolated picoliter droplets
}

\author{
N. Reginald Beer ${ }^{1 *}$, Elizabeth K. Wheeler ${ }^{1}$, Lorenna Lee-Houghton ${ }^{2}$, Nicholas Watkins ${ }^{3}$, \\ Shanavaz Nasarabadi ${ }^{1}$, Nicole Hebert ${ }^{4}$, Patrick Leung ${ }^{4}$, Don W. Arnold ${ }^{4}$, Christopher G. Bailey ${ }^{1}$, \\ and Bill W. Colston ${ }^{1}$ \\ ${ }^{1}$ Lawrence Livermore National Laboratory, 7000 East Avenue, Livermore, California 94551, \\ USA \\ ${ }^{2}$ Department of Biological Engineering, Massachusetts Institute of Technology, Cambridge, MA \\ 02139, USA \\ ${ }^{3}$ Department of Electrical and Computer Engineering, Purdue University, West Lafayette, IN \\ 47907, USA \\ ${ }^{4}$ Eksigent Technologies, Dublin, California 94568, USA
}

RECEIVED DATE (to be automatically inserted after your manuscript is accepted if required according to the journal that you are submitting your paper to)

*beer2@llnl.gov 
ABSTRACT. The first lab-on-chip system for picoliter droplet generation and RNA isolation, followed by reverse transcription, and PCR amplification with real-time fluorescence detection in the trapped droplets has been developed. The system utilized a shearing T-junction in a fused silica device to generate a stream of monodisperse picoliter-scale droplets that were isolated from the microfluidic channel walls and each other by the oil phase carrier. An off-chip valving system stopped the droplets on-chip, allowing thermal cycling for reverse transcription and subsequent PCR amplification without droplet motion. This combination of the established realtime reverse transcription-PCR assay with digital microfluidics is ideal for isolating single-copy RNA and virions from a complex environment, and will be useful in viral discovery and geneprofiling applications.

Keywords: on-chip PCR, picoliter PCR, microdroplet, digital microfluidics, real-time PCR, digital PCR, w/o emulsion, qPCR, reverse transcription PCR, RT-PCR, gene profiling

\section{Introduction}

The accurate detection and identification of low levels of pathogens in complex samples is critical in a number of applications such as clinical medicine, environmental monitoring, and biodefense. Developments in highly sensitive nucleic acid amplification techniques, as well as the explosion in the available genome sequences due to comprehensive sequencing efforts, have made PCR analysis the method of choice for low level pathogen detection. Taqman-based realtime quantitative PCR using dual-labeled fluorogenic probes to provide accurate quantitation of gene copies ${ }^{1}$ is now used extensively for rapid characterization of pathogens since it requires no post amplification manipulation or integration with electrophoresis systems, hybridization arrays, or mass spectrometry ${ }^{2}$. Also, with the addition of a reverse transcription step, PCR assays can be modified to enable detection of low levels of RNA. This capability, reverse transcription PCR 
(RT-PCR), is critical in the detection of emerging viral threats, many of which are caused by retroviruses, organisms having RNA genomes.

Recently, development of microfluidic techniques has led to advances in performing PCR assays including reduction of sample and reagent consumption, and increases in speed, sensitivity and dynamic range. Emulsion techniques are a promising new variation of microfluidics where aqueous droplets act as tiny, isolated chemical reactors suspended in a hydrophobic medium. This approach offers the possibility of greatly simplified microfluidic fabrication as well as increases in sample throughput. Emulsion PCR has been used to detect single genome copies and has been integral in new methods of high throughput genome sequencing ${ }^{3}$. Because of the segregation of DNA copies into individual compartments, amplification bias, where short or higher copy number sequences are preferentially amplified, is reduced. This advantage has been realized in medical diagnostics where low levels of mutant oncogenes ${ }^{4}$ and a series of Y chromosome microdeletions ${ }^{5}$ have been found that were not detectable using traditional bulk amplification methods. Such a capability is critical in detection of important, but low copy number pathogens whose presence could be masked by high concentration, interfering background species in complex environmental or clinical samples.

A complete analysis of samples containing pathogens requires the ability to detect both DNA and RNA based organisms. While extensive work has been done on microfluidic PCR based assays, less has been done on RT PCR systems. In microfabricated compartments, RT-PCR in $450 \mathrm{pL}$ reactors has been demonstrated down to an estimated level of 34 RNA copies ${ }^{6}$. Nanoliter volume RT-PCR has been integrated to on-chip capillary electrophoresis to detect 11 RNA copies in 45 minutes $^{7}$. RT-PCR has also been performed in batch-generated emulsions at the single copy level ${ }^{8}$. However, bulk emulsion methods typically use end-point amplification 
detection after breaking the emulsion and recombining all the amplified nucleic acids ${ }^{8}$. To perform rapid real-time detection the droplets must be focused into a channel so background fluorescence from droplets outside the focal plane does not affect the fluorescence intensity measurement, allowing optical interrogation of individual reactors. Additionally, generation of droplets in a microfluidic channel offers a level of control over size and quantity of reactants in droplets not achievable with bulk emulsion methods.

Previously, we demonstrated detection of single copies of the DNA virus vaccinia using microdroplet real time $\mathrm{PCR}^{9}$. Given that a number of recently emerging threats are retroviruses, we are interested in expanding our capabilities to include detection of low levels of RNA viruses as well. Here we report the first on-chip digital microfluidic real-time RT-PCR instrument for generating monodisperse isolated picoliter reactors and demonstrate its use in thermal cycling for reverse transcription and PCR, and real time detection of single genome copies.

\section{Experimental section}

Droplet Generation chip design A microfluidic chip with channels coated to create hydrophobic surfaces was used to create droplets and perform PCR amplification. Monodisperse droplets with tunable volumes are generated at a T-junction where a flowing oil stream shears off slugs of an aqueous stream into a main flow channel. The nascent aqueous boluses relax into a spherical shape provided the channel cross section is sufficiently large to prevent wall interactions.

Droplet size is adjusted by varying channel geometry, flow rate, and dispersed phase viscosity ${ }^{10}$

11. An effective T-junction width for channels of rectangular cross section was determined previously $^{9}$ and has been applied to the circular cross section used in this study. The width of the channels in the current device increases from $60 \mu \mathrm{m}$ at the T-junction to $150 \mu \mathrm{m}$ downstream to allow more droplets to be measured simultaneously in the fixed field of view. Two inlet and 
one outlet fluidic connections were made to the edge of the chip using either epoxied fused silica capillaries or a gasket assembly connector. To prevent cross-contamination between experiments, the fluid lines and channels were rinsed with a $10 \%(0.6 \% \mathrm{~m} / \mathrm{v}, 10 \mu \mathrm{L})$ solution of household bleach while the chip was heated to $90^{\circ} \mathrm{C}$, followed by deionized water $(120 \mu \mathrm{L})$ prior to each run.

Device fabrication Microfluidic chips were fabricated by isotropically etching $30 \mu \mathrm{m}$ deep features into two $1 \mathrm{~mm}$-thick fused silica wafers. The two etched wafers, which are mirror images of each other, were then aligned and thermally bonded, generating channels which are elliptical in cross section. To enhance detection of fluorescence from the droplets in the channels, the microfluidic devices were coated on one side with a $30 \mathrm{~nm}$ adhesion layer of chromium and a $300 \mathrm{~nm}$ layer of gold by an e-beam thermal evaporator, creating a mirror plane at the top surface of the chip. Prior to use, all chip-based devices and fused silica capillary tubing were rendered hydrophobic through coating with vapor-deposited $1 H, 1 H, 2 H, 2 H$ perfluorodecyltrichlorosilane (FDTS), performed by Applied Microstructures, Inc.

System Architecture In addition to the device redesign and the chip substrate change, the picoliter droplet real-time PCR instrument previously described ${ }^{9}$ was extensively modified for RT-PCR as follows. Flow for both the aqueous and oil streams was controlled individually by a NanoFlow Metering System (PN: 950-00008, Eksigent Technologies). A mixture of viral MS2 sample and RT-PCR master mix was delivered to the aqueous channel of the chip and PCR mineral oil (M8662, Sigma-Aldrich) to the oil channel. Stabilizing additives were not required, which simplified translation of the RT-PCR protocol to the picoliter regime. Two Rheodyne 
MHP7900-500-1 six-port valves with attached holding coils for the oil and aqueous phases were coupled to a Rheodyne MHP7980-500-1 six-port flow synchronization valve to facilitate droplet trapping on-chip. The Peltier Thermo Electric Cooler (TEC) used previously was replaced with the system described below in Thermal Cycling. Finally, an automated 2-axis microscope stage was implemented as described in Fluorescence detection below. See Figure 1a) \& b).

Thermal cycling The contact thermal cycling assembly was built using an $18.9 \mathrm{ohm}$ flexible Kapton resistive heating element in contact with an aluminum heat spreading strip on the opposite side of which a thermally conductive silicone pad was mounted to provide conformal contact with the device. To increase the cooling surface area and maximize the cooling rate, 0.001 in-thick copper foil was mounted to the aluminum heat spreading strip. House-generated $100 \mathrm{psi}$ compressed air was directed to the copper foil for device cooling. The temperature was controlled through a Watlow Series 96 temperature controller connected to a thermocouple attached to the aluminum heat spreading strip. The programmed RT-PCR profile was 15 minutes at $55^{\circ} \mathrm{C}$ for the virion capsid lysis and reverse transcription step, followed by $180 \mathrm{~s}$ at $95^{\circ} \mathrm{C}$, then 40 cycles of $94^{\circ} \mathrm{C}$ for $20 \mathrm{~s}, 55^{\circ} \mathrm{C}$ for $15 \mathrm{~s}$, and $69^{\circ} \mathrm{C}$ for $10 \mathrm{~s}$. The thermal profile was calibrated using a 0.003 in Type-K bare-wire thermocouple embedded into the microfluidic device, in plane with the microfluidic channel. The heating and cooling rates were $\sim 5^{\circ} \mathrm{C} / \mathrm{s}$ and $2^{\circ} \mathrm{C} / \mathrm{s}$, respectively.

PCR Reagents Per $25 \mu \mathrm{L}$, the RT-PCR mastermix contained 1x Superscript III reaction buffer, 4 $\mu \mathrm{L}$ of Superscript III Reverse Transcriptase / Platinum Taq Polymerase mix (Invitrogen), $0.2 \mu \mathrm{M}$ forward primer 5'-GGAGAGACAGGGCACTGCTA-3', $0.2 \mu \mathrm{M}$ reverse primer 5'- 
TTGGCCATACGGATTGTACC-3', $0.2 \mu \mathrm{M}$ 5'FAM-CCCAAATCTCAGCCATGCATCGAG3'BHQ1 probe (Biosearch Technologies), $0.04 \mu \mathrm{g} / \mu \mathrm{L}$ BSA, and MS2. The MS2 virions were cultured at Lawrence Livermore National Laboratory using standard procedures. Virion concentration was quantified by plaque assays to determine the initial stock titer of active virus particles in plaque forming units per $\mathrm{mL},(\mathrm{pfu} / \mathrm{mL})$. The stock template solution was used to generate a dilution series of $1: 1,10,100,500$, and 1000 for the on chip experiments.

Fluorescence detection Fluorescence was monitored using a Nikon TE2000-U microscope fitted with a 41001 FAM Chroma filter set, a 4× Nikon objective and a Nikon Intensilight DC arc lamp. Fluorescence images were captured at up to three imaging locations with a CoolSnap $\mathrm{HQ}^{2} \mathrm{CCD}$ (1392 x 1040 pixels) with a $100 \mathrm{~ms}$ acquisition time to avoid observable fluorophore photobleaching effects. Bright field images were acquired with a MotionPro HS-4 CMOS camera (Redlake) at 5,000 frames per second (512 x 512 pixels). The fluorescence microscope imaged droplets in a $750 \times 1,250 \mu \mathrm{m}$ section of the chip during the annealing phase of each cycle for real-time detection. Scanning between the imaging locations was performed by an ASI motion controller and XY stage mounted on the Nikon microscope and controlled in LabVIEW.

Data Analysis Brightfield images were analyzed using the watershed edge detection method ${ }^{12}$ to identify and locate the individual droplets. Average pixel intensity for each droplet was tracked through 40 cycles of PCR. The droplet real-time fluorescence curves were then processed using an algorithm adopted from a commercial instrument (SmartCycler Operator Manual D0190 Rev. D, Cepheid, Sunnyvale, CA) to subtract background fluorescence and determine cycle threshold, $\mathrm{C}_{\mathrm{t}}$, using a threshold value of 0.2 intensity units.

\section{Results and Discussion}


The device successfully generated and trapped highly monodisperse droplets on-chip for use as captive RT-PCR reactors. Adequate droplet spacing was used to ensure that flow stoppage caused no observable coalescence. Once stopped, the droplets remained stationary throughout the duration of PCR thermal cycling. A brightfield image of a typical trapped droplet field is shown in Figure 1a).

Sixteen separate experiments were performed using sample concentrations ranging from 0.05 to $47 \mathrm{pfu} /$ droplet. On-chip RT-PCR results from one of the three imaging locations for a typical run is summarized in Figure 2. For this particular run, droplets had an average diameter of 51 $\mu \mathrm{m}$ and a computed average droplet volume of $70 \mathrm{pL}$. Fluorescence images were recorded at each thermal cycle and images from two characteristic cycles, 0 and 30, are shown in Figure 2b)2c). As expected, fluorescence of the probe increases as amplification proceeds. Figure 2d) shows the droplet intensity real-time fluorescence curves generated from the fluorescence images for the same droplet field. The $\mathrm{C}_{\mathrm{t}}$, where the fluorescence intensity crosses the critical threshold, averaged 23.6 with a standard deviation of 2.1 for the run, as determined by the data analysis algorithm. The real-time fluorescence intensity curves for each droplet in the study exhibited the typical exponential, linear, and plateau phases of PCR, similar to those observed in bench-top microliter-scale reactions.

Analysis of fluorescence intensities of all droplets from all sixteen runs (1,241 total droplets) led to the cycle threshold versus log of copy number in pfu/droplet as shown in Figure 3. Repeatable amplification was observed at all positive starting concentrations, with the expected linear relationship between cycle threshold and log of starting copy number per droplet for starting concentrations at or above single copy levels. The trend in increasing cycle threshold with decreasing pfu/droplet plateaus at concentrations where copy number per droplet falls 
significantly below one (red points in Figure 3). This behavior is expected at dilutions where individual droplets either contain one or no RNA genomes, so lowering the concentration further does not lower the starting copy number for individual droplets, but will lower the overall number of droplets containing genomes.

Below approximately 1 pfu per droplet, the percentage of successfully amplified droplets falls appreciably, as seen in Figure 4, which plots the percent of droplets showing amplification versus the calculated pfu copy number per droplet. This decrease in percent amplification is consistent with the plateau of the $\mathrm{C}_{\mathrm{t}}$ seen in Figure 3, indicating digital PCR. The profile expected from Poisson statistics is shown as a blue line for comparison. The data exhibit the same general trend as the Poisson-predicted curve, but the observed droplet amplification percentages are higher than predicted by Poisson statistics. Such a shift in the Poisson curve from the data would be expected in the case where the plaque assay measurement was an under representation of the actual number of viral genomes present. This is consistent with previous studies, which have reported that viral titers determined by quantitative PCR tend to be at least 10 times higher than those measured by plaque assays ${ }^{13,14}$.

The agreement between observed and Poisson-predicted droplet amplification for the total starting copy concentration across all dilutions demonstrates the suitability of droplets for sensitive quantitative RT-PCR. Future directions of this research will focus on optimizing the assay for the picoliter scale emulsion, demonstrating this method on different viral genomes, and adapting to continuous flow thermal cycling ${ }^{15}$ to increase droplet throughput.

\section{Conclusions}


We have demonstrated on-chip RNA isolation in picoliter droplets, reverse transcription within the individual droplets, and subsequent real-time PCR with fluorescence detection of amplification in each interrogated droplet. The system employed a method of sample partitioning into monodisperse picoliter droplets emulsified in oil to generate isolated chemical reactors for high sensitivity nucleic acid detection. The method required approximately twentythree cycles for single-copy real-time reverse transcription from RNA, amplification, and detection on-chip using Taqman-based probes. The results show the method is well-suited to quantitative PCR applications, given the observability of Poisson statistics in the picodropletdiscretized sample. Applying digital microfluidics to real-time RT-PCR combines the advantages of on-chip processing of picoliter reactors with single-copy detection.

\section{References}

(1) Heid, C. A.; Stevens, J.; Livak, K. J.; Williams, P. M. Genome Res. 1996, 6, 986-994.

(2) Belgrader, P.; Benett, W.; Hadley, D.; Richards, J.; Stratton, P.; Mariella, R.; Milanovich, F. Science 1999, 284, 449-450.

(3) Margulies, M.; Egholm, M.; Altman, W. E.; Attiya, S.; Bader, J. S.; Bemben, L. A.; Berka, J.; Braverman, M. S.; Chen, Y. J.; Chen, Z. T.; Dewell, S. B.; Du, L.; Fierro, J. M.; Gomes, X. V.; Godwin, B. C.; He, W.; Helgesen, S.; Ho, C. H.; Irzyk, G. P.; Jando, S. C.; Alenquer, M. L. I.; Jarvie, T. P.; Jirage, K. B.; Kim, J. B.; Knight, J. R.; Lanza, J. R.; Leamon, J. H.; Lefkowitz, S. M.; Lei, M.; Li, J.; Lohman, K. L.; Lu, H.; Makhijani, V. B.; McDade, K. E.; McKenna, M. P.; Myers, E. W.; Nickerson, E.; Nobile, J. R.; Plant, R.; Puc, B. P.; Ronan, M. T.; Roth, G. T.; Sarkis, G. J.; Simons, J. F.; Simpson, J. W.; Srinivasan, M.; Tartaro, K. R.; Tomasz, A.; Vogt, K. A.; Volkmer, G. A.; Wang, S. H.; Wang, Y.; Weiner, M. P.; Yu, P. G.; Begley, R. F.; Rothberg, J. M. Nature 2005, 437, 376-380.

(4) Vogelstein, B.; Kinzler, K. W. Proc. Natl. Acad. Sci. U.S.A. 1999, 96, 9236-9241.

(5) Ge, Q.; Liu, Z.; Bai, Y.; Zhang, D.; Yu, P.; Lu, Z. Anal. Biochem. 2007, 367, 173-178.

(6) Marcus, J. S.; Anderson, W. F.; Quake, S. R. Anal. Chem. 2006, 78, 956-958.

(7) Toriello, N. M.; Liu, C. N.; Mathies, R. A. Anal. Chem. 2006, 78, 7997-8003.

(8) Nakano, M.; Nakai, N.; Kurita, H.; Komatsu, J.; Takashima, K.; Katsura, S.; Mizuno, A. J. Biosci. Bioeng. 2005, 99, 293-295.

(9) Beer, N. R.; Hindson, B. J.; Wheeler, E. K.; Hall, S. B.; Rose, K. A.; Kennedy, I. M.; Colston, B. W. Anal. Chem. 2007. 
(10) Thorsen, T.; Roberts, R. W.; Arnold, F. H.; Quake, S. R. Phys. Rev. Lett. 2001, 86, 4163-4166.

(11) Cristini, V.; Tan, Y. C. Lab Chip 2004, 4, 257-264.

(12) Gonzalez, R. C., Woods, R.E. Digital Image Processing, 2nd Edition ed.; Prentice Hall, 2002.

(13) Toriniwa, H.; Komiya, T. Microbio. Immun. 2006, 50, 379-387.

(14) Perkins, S. M.; Webb, D. L.; Torrance, S. A.; El Saleeby, C.; Harrison, L. M.; Aitken, J. A.; Patel, A.; DeVincenzo, J. P. J. Clin. Microbio. 2005, 43, 2356-2362.

(15) Kopp, M. U.; de Mello, A. J.; Manz, A. Science 1998, 280, 1046-1048.

Acknowledgements. We thank John Breneman, James Frank, Joshua Deotte, and Kelly Spruiell for technical assistance. This work performed under the auspices of the U.S. Department of Energy by Lawrence Livermore National Laboratory under Contract DE-AC5207NA27344. The project \#06-ERD-064 was funded by the Laboratory Directed Research and Development Program at LLNL. 
Figure 1. On-chip RT-PCR device and schematic. Panel a) shows the fused silica device with an inset of monodisperse $\sim 70 \mathrm{pL}$ droplets trapped and ready for subsequent PCR. Panel b) is a schematic of the instrument. 
Figure 2. Representative on-chip RT-PCR results from one of the three imaging locations. Panel a) shows the brightfield image after thermal cycling. Droplets have a volume of $\sim 70 \mathrm{pL}$ Panels b) \& c) show the fluorescence intensity at cycles 0 and 30 respectively. Panel d) is a plot of fluorescence intensity versus cycle number for all the droplets in the field of view- with a 5-point moving average applied to the intensities to smooth the real-time curve. Note that 23 of the 25 droplets in panel a) supported amplification. 
Figure 3. Cycle Threshold versus $\log$ (plaque forming units per droplet) for all sixteen runs. The total number of droplets analyzed is 1241 . Error bars correspond to $+/-$ standard deviation. The plateau at the lower concentrations (points in red) signifies the single-copy $\mathrm{C}_{t}$ limit has been reached. The $\mathrm{R}^{2}$ value of $\sim 0.92$ is for the range of -0.55 to 1.70 . 
Figure 4. Percentage of droplets amplified versus starting copy number per droplet (symbols) against that predicted by Poisson statistics (blue curve). 
(a)

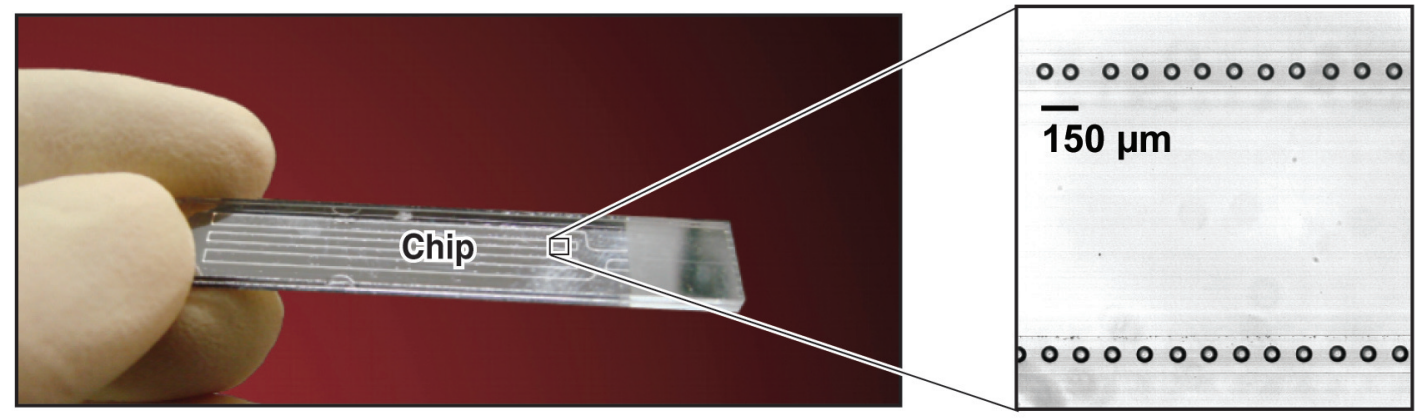

(b)

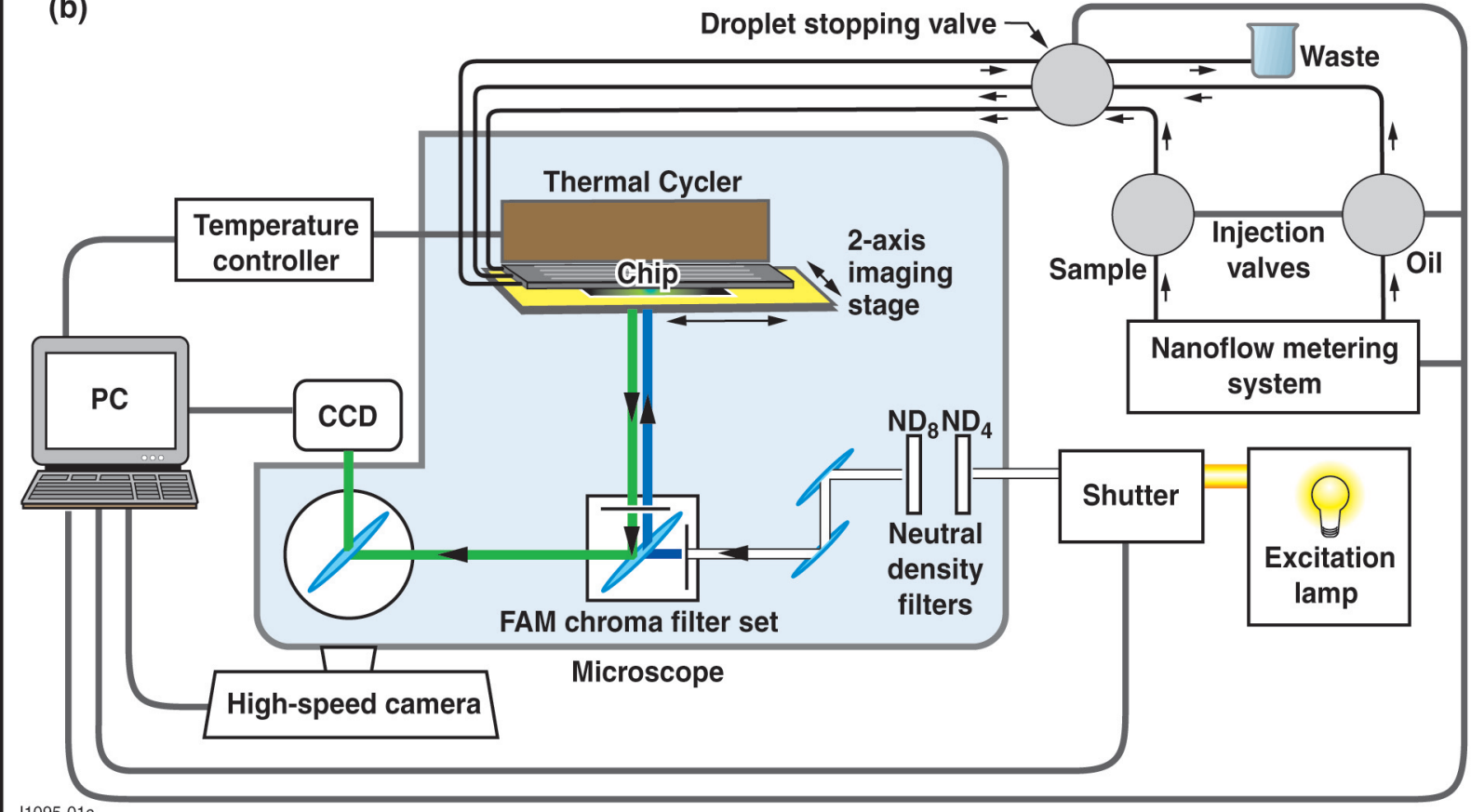


(a) Brightfield

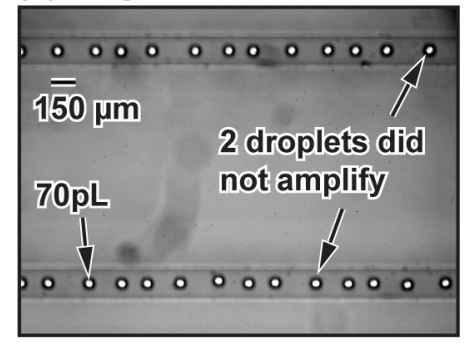

(b) Cycle 0

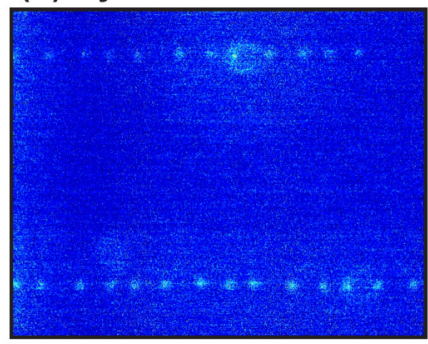

(c) Cycle 30

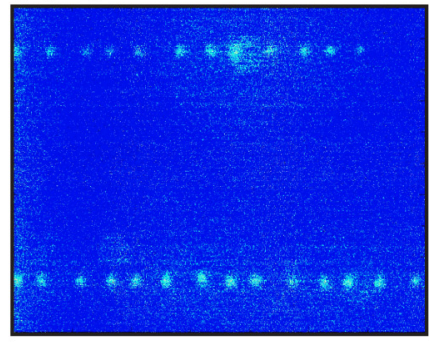

(d)

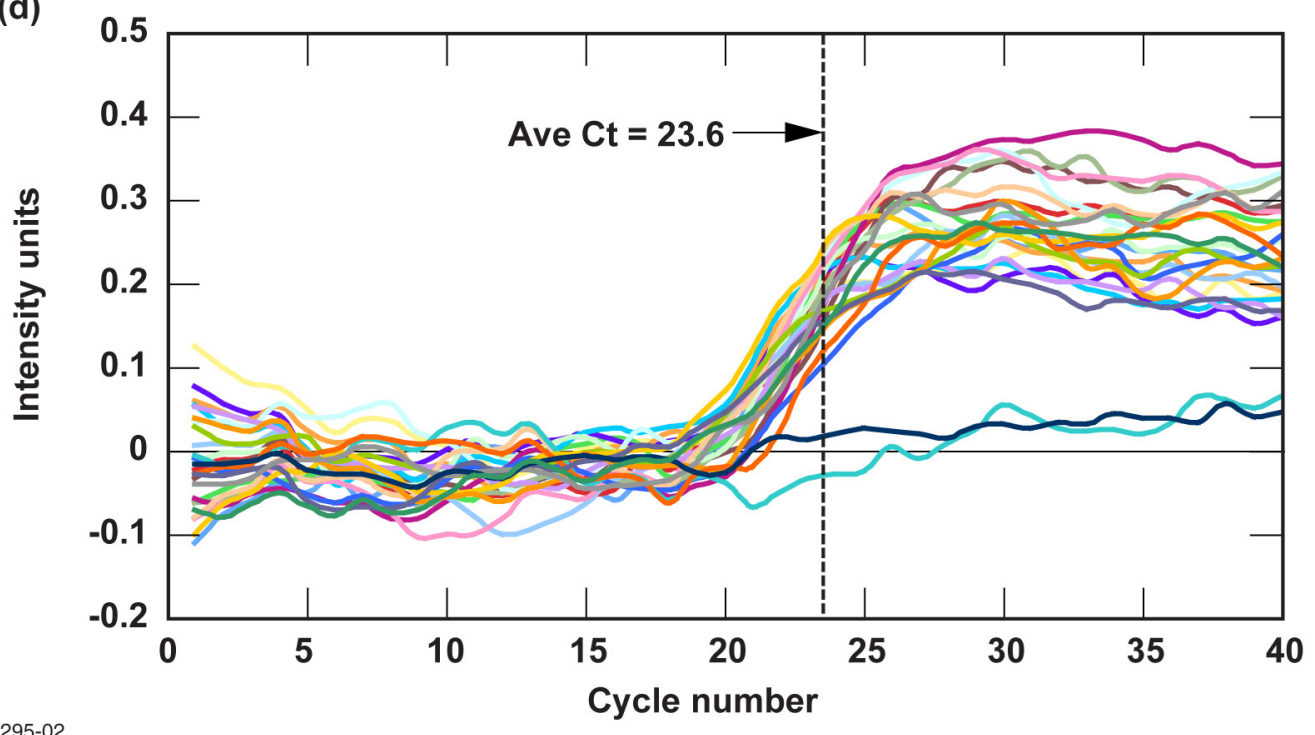




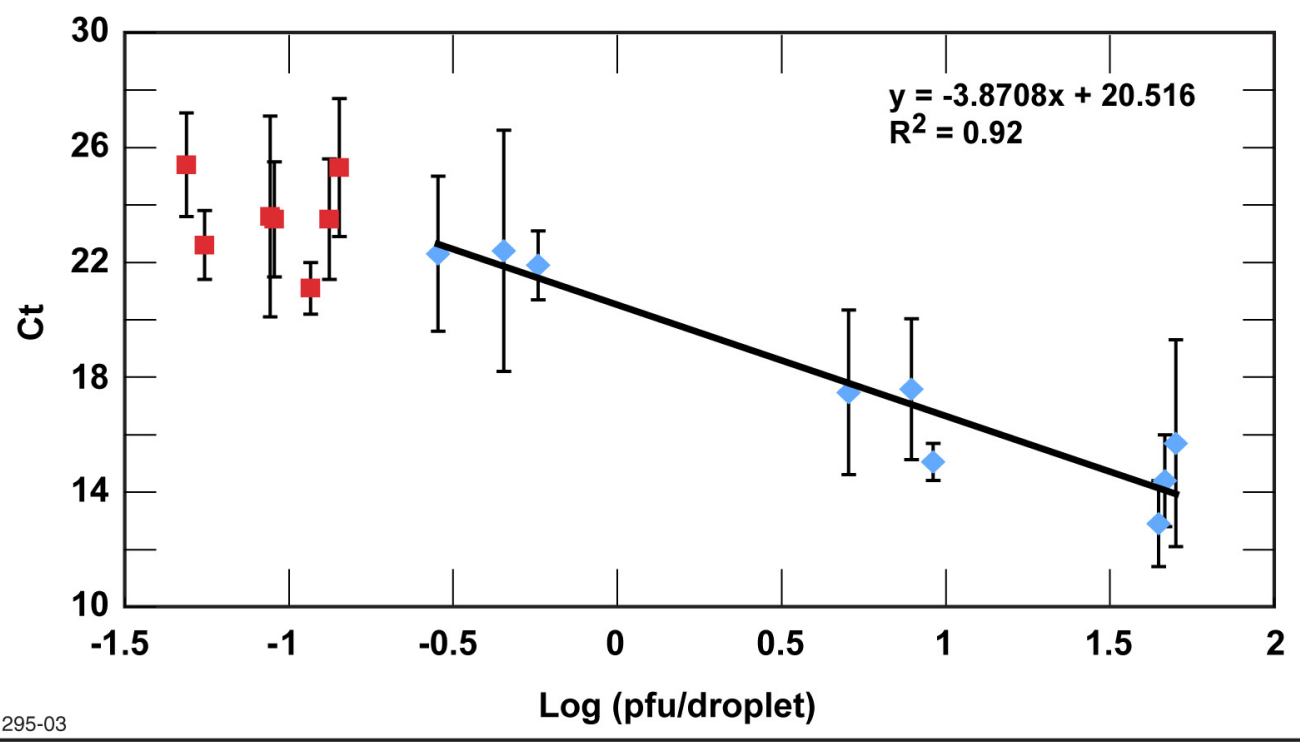




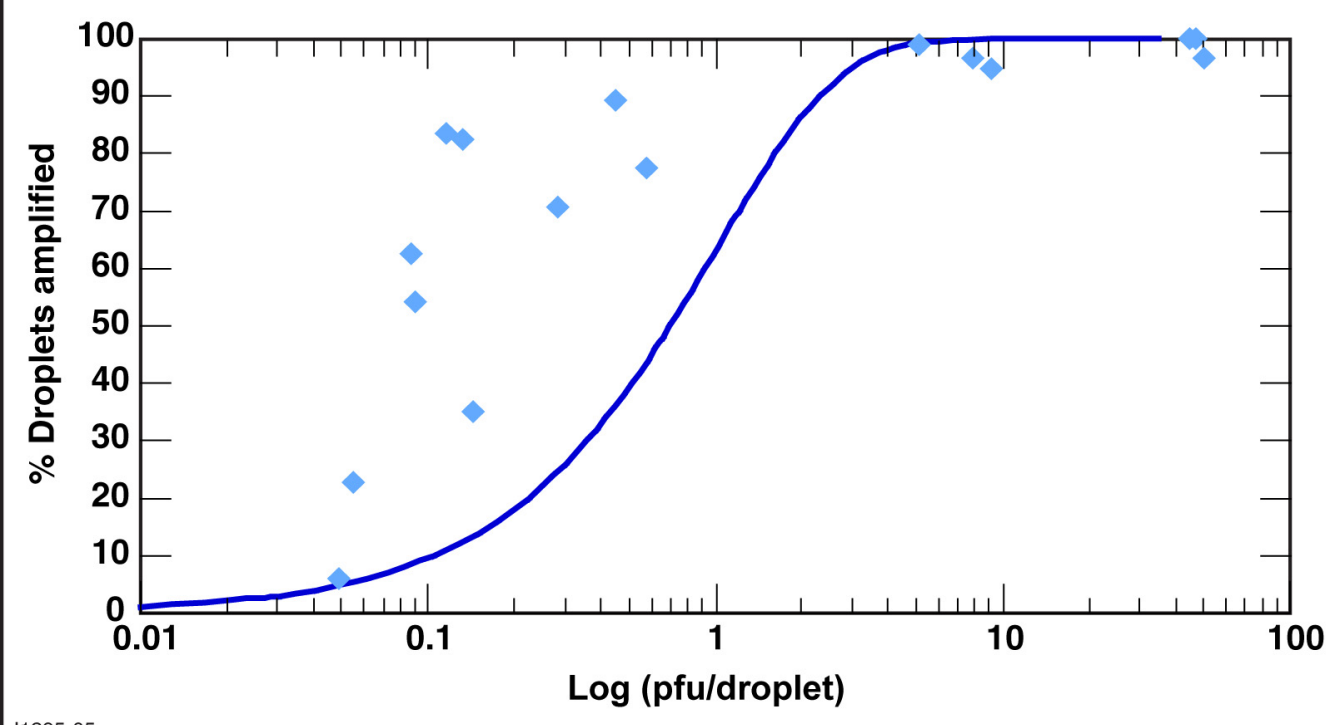

